\title{
Laparoscopic management of endometriosis in infertile women and outcome
}

\author{
Latika Sahu*, Anjali Tempe
}

\begin{abstract}
Department of Obstetrics \& Gynecology, Maulana Azad Medical College \& associated Lok Nayak Hospital, New Delhi-110002, India
\end{abstract}

Received: 25 February 2013

Accepted: 22 March 2013

\section{* Correspondence:}

Dr. Latika Sahu,

E-mail: latikasahu@gmail.com

(C) 2013 Sahu L et al. This is an open-access article distributed under the terms of the Creative Commons Attribution License, which permits unrestricted use, distribution, and reproduction in any medium, provided the original work is properly cited.

\begin{abstract}
Background: The prevalence of endometriosis is estimated to be $\sim 3 \%$ in women of reproductive age and it is observed in 20 to $70 \%$ of patients presenting with infertility. For patients presenting with moderate or severe endometriosis, the fertility results were better with surgical treatment than with no treatment or with medical treatment. The objective of this study was to identify endometriosis in infertile women during diagnostic laparoscopy, to demonstrate the surgical feasibility and outcome of laparoscopic management of endometriosis in infertile women.

Methods: Endometriosis patients with infertility were analyzed. When operating on patients of endometriosis following aspects of surgery were correlated with pregnancy outcome. Endometrioma removal by cystectomy/ drainage and fulguration, conservation of ovarian tissue, restoration of tubo-ovarian relationship, adhesiolysis.

Results: There were 191 cases of infertility for which diagnostic laparoscopy were performed for the period of 2 years. Only 13 cases $(6.8 \%)$ had features of moderate/severe endometriosis. $4(30.76 \%)$ cases had severe and $9(69.24 \%)$ cases had moderate endometriosis. All cases were treated by laparoscopic surgery. $6(47.04 \%)$ of them conceived. $5(83.4 \%)$ of them conceived with IUI and one patient (16.6\%) conceived with IVF treatment.

Conclusions: The use of laparoscopic surgery in the treatment of moderate/severe endometriosis improves success rates of IUI \& IVF.
\end{abstract}

Keywords: Endometriosis, Laparoscopic surgery, Infertile

\section{INTRODUCTION}

The prevalence of endometriosis is estimated to be $\sim 3 \%$ in women of reproductive age and it is observed in 20 to $70 \%$ of patients presenting with infertility. ${ }^{1}$ At present it is generally agreed that surgical treatment is the treatment of choice for infertile patients presenting with endometriosis. For patients presenting with moderate or severe endometriosis, the fertility result are better with surgical treatment than with no treatment or with medical treatment. ${ }^{2}$
When fertility surgery is indicated, operative laparoscopy results in outcomes as good as those from similar procedures performed via open laparotomy and is associated with a shorter hospital stay, lower incidence of ileus, and faster recovery. In addition, there is less contamination of the surgical field with glove powder or lint, bleeding is reduced due to tamponade of small vessels by the pneumoperitoneum, and drying of tissues is minimal because surgery occurs in a closed environment. All of these factors contribute to reduce postoperative adhesion formation and its associated morbidity (e.g., pain, impaired fertility, bowel obstruction). 
In the present study we are finding mainly the fertility outcome of the infertile women with moderate/ severe endometriosis operated with laparoscopic surgeries.

\section{METHODS}

This is a prospective observational study carried out in the department of Obstetrics \& Gynecology \& IVF \& Reproductive biology center of Maulana Azad Medical College \& associated Lok Nayak hospital, New Delhi for two years. All the infertile women attended the center under our unit were investigated for infertility including transvaginal ultrasonography and diagnostic laparoscopy $\&$ those who were found to be moderate/severe endometriosis were operated with laparoscopic surgeries. All the patient included in the study had no associated male infertility. When operating on patients of endometriosis following aspects of surgery were correlated with pregnancy outcome-endometrioma removal by cystectomy/ drainage and fulguration, conservation of ovarian tissue, restoration of tubo-ovarian relationship, adhesiolysis. Fertility outcome of the patients with endometriosis were noted.
In all cases the dissection was carried out using laparoscopic scissors and haemostasis was always performed with bipolar coagulation. All endometriotic lesions were treated in all cases during the same operative procedure. During the initial diagnostic phase of the laparoscopy, the severity of the endometriotic lesions was categorized using the American Society for Reproductive Medicine revised classification system for endometriosis (ASRM 1996). Endometriosis was defined histologically by the presence of ectopic glands together with stroma.

Infertility was defined as at least 12 months of unprotected intercourse without resulting in pregnancy. Endometriosis was confirmed by positive histological result.

\section{RESULTS}

Moderate \& severe endometriosis was found in 13 out of 191 infertile women $(6.8 \%) .4(30.76 \%)$ cases had severe and $9(69.24 \%)$ cases had moderate endometriosis.

Table 1: Demographic characteristics of women undergoing laparoscopic surgery for endometriosis.

\begin{tabular}{|lll|}
\hline Characteristic & No. of Patients & Percentage \\
\hline 1. Age- in years & 1 & 7.7 \\
\hline $20-25$ yr & 4 & 30.8 \\
\hline $25-30$ yr & 5 & 38.5 \\
\hline $30-35$ yr & 3 & 23.0 \\
\hline $35-40$ yr & & \\
\hline 2. Socio-economic status & 7 & 53.8 \\
\hline Lower middle class & 5 & 38.5 \\
\hline Upper middle class & 1 & 7.7 \\
\hline Upper class & & \\
\hline 3. Habitat & 8 & 61.6 \\
\hline Urban & 3 & 23.0 \\
\hline Urban-slum & 2 & 15.4 \\
\hline Rural & & \\
\hline 4. Type of infertility & 11 & 84.6 \\
\hline Primary & 2 & 15.4 \\
\hline Secondary & & \\
\hline 5. Diagnosed years following married life & 2 & $15.4 \%$ \\
\hline $1-5$ yr & 3 & 23.0 \\
\hline $5-10$ yr & 6 & 46.2 \\
\hline $10-15$ yr & 2 & $15.4 \%$ \\
\hline$>15$ yr & & \\
\hline
\end{tabular}

Table 1 shows the demographic characteristics of women undergoing laparoscopic surgery for endometriosis. The incidence increases with age. $61.5 \%$ patients were in the age group of 30-40 years. Most of our cases had come after more than 10 years of married life. $92.3 \%$ cases were from middle class socio-economic group. Most of them belong to primary infertility $(84.6 \%)$. Secondary infertility cases were mostly following an abortion. Most of the cases were from urban area. 
Table 2: Laparoscopic findings of women with endometriosis.

\begin{tabular}{|lll|}
\hline Characteristic Findings & No. of Patients & Percentage \\
\hline 1. Site of endometriosis & & $100 \%$ \\
\hline Ovary- & 13 & 46.2 \\
\hline Unilateral & 6 & 53.2 \\
\hline Bilateral & 7 & 84.6 \\
\hline POD & 11 & $15.4 \%$ \\
\hline Posterior surface of uterus & 2 & 7.7 \\
\hline Uterosacral ligaments & 1 & \\
\hline 2. Adhesions & & 84.6 \\
\hline Present & 11 & 15.4 \\
\hline Absent & 2 & $61.6 \%$ \\
\hline 3. Chromopertubation & & $51.4 \%$ \\
\hline Bilateral patent tubes & 8 & $51.4 \%$ \\
\hline Unilateral patent tube & 2 & $7.6 \%$ \\
\hline Bilateral tubal block & 2 & \\
\hline Unilateral tubal block & 1 & $23.0 \%$ \\
\hline 4. Associated lesions & & $7.6 \%$ \\
\hline Fibroid & 3 & $38.5 \%$ \\
\hline Hydrosalpinx & 1 & \\
\hline Polycystic ovary & 5 & \\
\hline
\end{tabular}

Table 2 shows Laparoscopic findings of women with endometriosis. All cases had endometriomas of ovary. Both ovaries were involved in $53.8 \%$ cases.77 \% had patent tubes and $23 \%$ had blocked tubes. $84.6 \%$ patient had peritoneal adhesions. According to the American
Society for Reproductive Medicine revised classification system for endometriosis (ASRM 1996) criteria, 4 $(30.76 \%)$ cases had severe and $9(69.24 \%)$ cases had moderate endometriosis.

Table 3: Methods of laparoscopic surgical procedures done for the patients with endometriosis.

\begin{tabular}{|lll|}
\hline Surgical Procedures & No. of Patients & Percentage \\
\hline 1. Endometrioma removal by cystectomy & 9 & $69.2 \%$ \\
\hline 2. Endometrioma Drainage and fulguration & 4 & $30.8 \%$ \\
\hline 3. Adhesiolysis & 11 & $84.6 \%$ \\
\hline
\end{tabular}

Table 3 shows the methods of laparoscopic surgical procedures done for the patients with endometriosis. Endometrioma removal by cystectomy done in $69.2 \%$ of cases. Adhesiolysis done in all cases where present and tubo-ovarian relationship was maintained following adhesiolysis. All cases were given injection Leuprolide $3.75 \mathrm{mg}$ monthly injection immediately following laparoscopic surgery. The patients were seen in consultation every month after operation. Then they were planned for ovulation induction \&IUI or IVF.

Table 4 shows the fertility outcome of the operated endometriosis patients.76.9\% of endometriosis treated cases ovulated with ovulation induction and 6 out of 10 patients conceived. IVF done for 3 patients and one of them conceived. IUI done for 7 patients and 5 of them conceived. All of them conceived within 6 months of surgery.

Five conceived patients had uneventful antenatal period with intense monitoring and delivered at term. Those who conceived with IUI had vaginal delivery following labor induction at term and the patient who conceived with IVF delivered by elective cesarean section with uneventful postoperative period.All five patients went home with healthy baby. One patient who conceived after IUI had spontaneous first trimester abortion. 
Table 4: Fertility outcome of the operated endometriosis patients.

\begin{tabular}{|lll|}
\hline $\begin{array}{l}\text { Fertility Outcome } \\
\text { 1. Ovulation occurred with } \\
\text { induction }\end{array}$ & No. of Patients & Percentage \\
\hline With clomiphene & $\mathbf{1 0}$ & $\mathbf{7 6 . 9 \%}$ \\
\hline With gonadotrophins & 1 & $7.7 \%$ \\
\hline With clomiphene \& gonadotrophins & 2 & $15.4 \%$ \\
\hline 2. Conceived & 7 & $53.8 \%$ \\
\hline With- & 6 & $46.0 \%$ \\
\hline With IUI & & $38.4 \%$ \\
\hline With IVF & 5 (Out of 7) & $7.7 \%$ \\
\hline 3. pregnancy outcome & 1 (Out of 3) & \\
\hline Abortion & & $7.7 \%$ \\
\hline Term pregnancy & 1 & $38.4 \%$ \\
\hline 4. Take home baby & 5 & $\mathbf{3 8 . 4 \%}$ \\
\hline
\end{tabular}

\section{DISCUSSION}

Endometriosis is a difficult gynecological disease to treat and sometimes to diagnose. Laparoscopy \& histological biopsy is the best way to confirm it. Ultrasound is useful for supporting the clinical diagnosis of endometrioma, but of limited value for diagnosis or determining extent of endometriosis since it lacks adequate resolution for visualizing adhesions and superficial peritoneal/ovarian implants. However, when there are sonographic signs suggestive of endometriomas, it is likely that moderate to severe endometriosis is present. ${ }^{3}$ Transvaginal ultrasound is a useful tool both to make and to exclude the diagnosis of an ovarian endometrioma. Visual inspection of the pelvis at laparoscopy is the gold standard investigation for diagnosis of endometriosis.

Since development of laparoscopic surgery in early 80's, there has been a considerable interest to manage endometriosis laparoscopically. Recently laparoscopic total pelvic peritoneal excision in 100 patient with different stages of the disease, has been shown in experienced hands to be safe and effective especially in preventing disease recurrence. $^{4}$

Laparoscopic treatment of endometriosis involves destroying the endometriotic lesions using electrosurgical desiccation or fulguration or by laser evaporation for minimal to mild disease while lysis of adhesions and excision of deep fibrotic lesions, enucleation of endometrioma may have to be performed for more severe forms of the disease.

Operative laparoscopy offers several advantages to laparotomy, primarily because of better visualization, less tissue trauma, and much shorter recovery time. The guiding surgical principle is complete removal of all endometriosis lesions, fibrosis, and adhesions, including those requiring deep dissection.
Analysis of the fertility results according to the means of surgical treatment of endometriosis-associated infertility showed no significant difference between laparoscopy and laparotomy. ${ }^{2}$

A study by Charles Chapron et al showed with classic treatment for endometriosis (e.g. lysis, cystectomy, bipolar coagulation of superficial peritoneal endometriotic lesions) with resection of deep endometriotic lesions infiltrating the USL. The overall intrauterine pregnancy (IUP) rate, including births and miscarriages, was $50.0 \%$ (15 patients). A total of 11 IUP occurred spontaneously. Three patients were pregnant with the use of ovulation induction, following dysovulation associated with endometriosis. One pregnancy was achieved after in-vitro fertilization (IVF). Of these 15 IUP, 12 patients gave birth normally at term, and three had an early miscarriage. None of the patients had an ectopic pregnancy. 5

Fuchs F, et al retrospectively analyzed 64 patients with infertility and having stage I - IV on laparoscopy. All treated laparoscopically but 20 were then excluded from the study (reason unspecified). 65\% (22 patients) got pregnant within 8.5 months. $89 \%$ with those with stage III \& 56\% with stage III-IV got pregnant within one month post-surgery. They recommend complete laparoscopic surgical treatment for such patients to increase their chance of pregnancy either spontaneously or with assisted reproductive techniques. ${ }^{6}$

Littman E, et al: A series of 29 patients with previously failed IVF treatment had laparoscopic evaluation $\&$ treatment by the same surgeon. 22 conceived including 15 non-IVF conception \& 7 after IVF treatment. Their conclusion was that in the absence of tubal occlusion \& male factor infertility, laparoscopic surgery may still to be considered for treatment of endometriosis even after multiple IVF treatment failure. ${ }^{7}$ 
A randomized clinical trial of two laparoscopic treatments of endometriomas: cystectomy versus drainage and coagulation, by Beretta $\mathrm{P}$ et al concluded that, for the treatment of ovarian endometriomas, a better outcome with a similar rate of complications is achieved with laparoscopic cystectomy than with drainage and coagulation. $^{8}$

A study by Hemmings $\mathrm{R}$ et al on results of laparoscopic treatments of ovarian endometriomas: laparoscopic ovarian fenestration and coagulation, concluded that Laparoscopic ovarian fenestration and coagulation of endometriomas leads to faster conception than ovarian cystectomy by laparotomy. Laparoscopic ovarian fenestration and coagulation of endometriomas is associated with cumulative clinical PRs and recurrence rates over 36 months that are similar to those associated with ovarian cystectomy. ${ }^{9}$

A systematic review and meta-analysis on the effect of surgical treatment for endometrioma on in vitro fertilization outcomes by Tsoumpou I, et al concluded that- Collectively the available data in the literature show that surgical management of endometriomas has no significant effect on IVF pregnancy rates and ovarian response to stimulation compared with no treatment. Randomized controlled trials are needed before producing best-practice recommendations on this topic. ${ }^{10}$

In our observational study the results were encouraging. Which shows that in a context of infertility it is reasonable to treat for moderate and severe endometriosis by laparoscopic surgical procedures to improve fertility outcome.

\section{CONCLUSIONS}

Every effort should be made to treat endometriosis actively at first laparoscopy with aim of complete removal of all visible lesions. There is good evidence that in experienced hands laparoscopic surgery has a lot to offer in infertile women with moderate to severe endometriosis. Though surgical treatment is technically demanding but patient rewarding in enhancing fertility.
Funding: No funding sources

Competing interests: None declared

Ethical approval: The study was approved by the Institutional Ethics Committee

\section{REFERENCES}

1. Matorras R, Rodriqez F, Pijoan JI, et al. Epidemiology of endometriosis in infertile women. Fertil Steril 1995;63:34-8.

2. Adamson GD, Pasta DJ. Surgical treatment of endometriosis-associated infertility: Meta-analysis compared with survival analysis. Am J Obstet Gynecol 1994;171:1488-1505.

3. Bulun SE. Endometriosis. N Engl J Med 2009; 360:268.

4. Trehan AK. Personal series, Minimal Access surgery unit, Dewsbury District Hosp, UK 2007.

5. Chapron C, Fritel X, Dubuisson JB. Fertility after laparoscopic management of deep endometriosis infiltrating the uterosacral ligaments. Hum Reprod 1999;14:329-32.

6. Fuchs F, Ravnal P, Salama S, et al. Reproductive outcome after laparoscopic treatment in an infertile population. J Gynaecol Obstet Biol Reprod (Paris) 2007;36:162-72.

7. Littman E, Gludice L, Lathi R, et al. Role of Laparoscopic treatment of endometriosis in patients with failed In vitro Fertilization population. Fertil Steril 2005;84:1574-8.

8. Beretta P, Franchi M, Ghezzi F, Busacca M, Zupi E, Bolis P. Randomized clinical trial of two laparoscopic treatments of endometriomas: cystectomy versus drainage and coagulation. Fertil Steril 1998;70:1176-80.

9. Hemmings R, Bissonnette F, Bouzayen R. Results of laparoscopic treatments of ovarian endometriomas: laparoscopic ovarian fenestration and coagulation. Fertil Steril 1998;70:527-9.

10. Tsoumpou I, Kyrgiou M, Gelbaya TA, Nardo LG. The effect of surgical treatment for endometrioma on in vitro fertilization outcomes: a systematic review and meta-analysis. Fertil Steril 2009;92:75-87.

DOI: $10.5455 / 2320-1770$. ijrcog20130613

Cite this article as: Sahu L, Tempe A. Laparoscopic management of endometriosis in infertile women and outcome. Int $\mathrm{J}$ Reprod Contracept Obstet Gynecol 2013;2:177-81. 